\title{
Diagnóstico de errores innatos del metabolismo
}

\author{
ERNA RAIMANN B. ${ }^{1}$ \\ 1. Pediatra, Prof. Asociado, INTA-Universidad de Chile, Director Médico CEDINTA, Universidad de Chile.
}

Palabras clave: Metabolismo, errores, trastorno, neonato, enzima

Los errores innatos del metabolismo son enfermedades monogénicas, de herencia autosómica recesiva en su mayoría. La alteración en un gen produce un defecto enzimático, que conduce a las alteraciones bioquímicas características de cada enfermedad metabólica y son responsables de los fenotipos desadaptativos propios de cada patología.

La mayoría de los errores innatos del metabolismo se manifiesta en la edad pediátrica, desde las primeras horas de vida y hasta la adolescencia, con síntomas y signos similares a otras patologías, no reconocerlas conduce a sequelas importantes siendo las más frecuentes: desnutrición, convulsiones y retardo mental. La prevención de estas secuelas con un diagnóstico oportuno es el enorme desafío al que se enfrentan los pediatras. Con este objetivo es necesario que los clínicos estén familiarizados con los síntomas y signos más comunes en los errores innatos del metabolismo, para poder iniciar oportunamente el tratamiento.

La aparición de la sintomatología puede ser muy aguda en el período neonatal ocasionando una emergencia clínica. En etapas posteriores de la vida, en cambio, la presentación suele ser menos aguda. Esto no significa, sin embargo, que las secuelas neurológicas y nutricionales sean menores.

Se puede dividir la presentación clínica de los errores innatos del metabolismo en 4 grandes grupos:

\section{Síntomas agudos en el período neonatal}

El recién nacido tiene un repertorio limitado de respuestas frente a enfermedades graves y los síntomas inespecíficos iniciales incluyen rechazo de la alimentación, vómitos explosivos, progresivo compromiso de conciencia, que comienza con letargia y somnolencia y termina en coma profundo, convulsiones, compromiso hemodinámico y muerte. Se puede encontrar también edema cerebral e incluso hemorragia intracraneana. Si bien un niño con sepsis presenta síntomas similares, debemos destacar que los pacientes con errores innatos del metabolismo también tienen un mayor riesgo de presentar una infección; por lo tanto esta sepsis puede enmascarar un error innato del metabolismo impidiendo un diagnóstico oportuno. Recomendamos por lo tanto siempre tomar muestras de sangre, orina y en tarjeta de papel filtro cuando exista la sospecha de un error innato del metabolismo.

Cuando el rechazo alimentario es menos evidente el niño puede debutar con apnea o con distress respiratorio. Las apneas en este caso son de origen central. La taquipnea, en cambio, puede ser reflejo de una acidosis metabólica como ocurre en las acidurias orgánicas. Los niños con defectos del ciclo de la urea, en el desarrollo inicial de un coma hiperamonémico, presentan hiperventilación debida al edema ce-

Correspondencia a:

Erna Raimann B.

E-mail: eraimann@inta.cl 
rebral y conduce a una alcalosis respiratoria, pues no se acumulan metabolitos que disminuyen el $\mathrm{pH}$.

Los vómitos son muy frecuentes en los errores innatos del metabolismo de las proteínas, aunque son más frecuentes en niños mayores que en recién nacidos.

Los síntomas son el resultado de los efectos tóxicos de los metabolitos que se acumulan en el sistema nervioso central. Estas sustancias durante el embarazo, cruzan la placenta y son eliminadas por la madre, por lo tanto el niño es sano al nacer. Las enfermedades que se manifiestan con mayor frecuencia de este modo en nuestra experiencia son: enfermedad de la orina olor a jarabe de arce, acidurias orgánicas, alteraciones del ciclo de la urea y galactosemia. El pediatra debe tener presente qué exámenes de laboratorio son fundamentales para esclarecer el diagnóstico, puesto que entre todas las enfermedades descritas los signos y síntomas tienen muy pequeñas variaciones (tabla 1).

El diagnóstico se puede confirmar cuantificando aminoácidos, ácidos orgánicos, ácido orótico, midiendo carnitina total y libre o acilcarnitinas por Espectrometría de Masas en Tandem, exámenes que deben ser referidos rápidamente al laboratorio especializado.

\section{Síntomas agudos y recurrentes de inicio más tardío}

Aproximadamente un tercio de los pacientes con enfermedades metabólicas tienen una presentación más tardía. El período libre de síntomas suele ser de 6 meses, al suspender la lactancia materna; después del 1 año o extenderse hasta etapas posteriores, incluyendo adolescentes o adultos jóvenes. Cada episodio puede derivar en mejoría espontánea o en muerte inexplicada. Durante el período entre las crisis el paciente parece normal. El inicio agudo se desencadena por una enfermedad infecciosa, fiebre, constipación o ingestas excesivas de proteínas. El catabolismo proteico también podría precipitar una descompensación. Las enfermedades son las mismas de presentación neonatal aguda, debiendo agregar aquí los defectos de oxidación de ácidos grasos.

En todo niño que presenta compromiso de conciencia se debe descartar una enfermedad

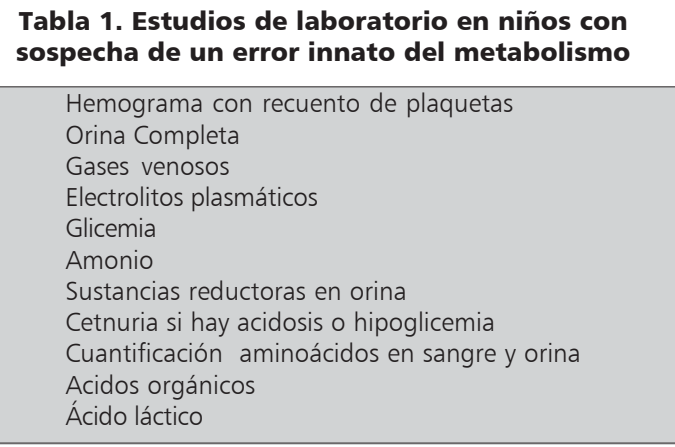

metabólica. Frente a diagnósticos como encefalitis, jaqueca o intoxicación se debe plantear un error innato del metabolismo, especialmente cuando el paciente presenta quetoacidosis, hiperamonemia o aumento del ácido láctico. En la presentación clínica predomina el coma con acidosis metabólica, con preponderancia de hiperamonemia o de hipoglicemia. Se acompaña de un amplio espectro de signos hepáticos, digestivos, neurológicos o psiquiátricos.

La hipoglicemia se presenta en enfermedades con intolerancia a las proteínas, pero es más frecuente en las alteraciones del metabolismo de los hidratos de carbono o de los ácidos grasos. Las glicogenosis cursan con hipoglicemia debida al bloqueo en la liberacion de glucosa desde glicógeno y es mayor en el ayuno. Los síntomas y signos son hipoglicemia, hepatomegalia y acidosis láctica.

La hipoglicemia puede ser muy importante en galactosemia y en intolerancia hereditaria a la fructosa, aunque los síntomas sólo aparecen después de ingerir estos azúcares.

Los defectos de oxidación de ácidos grasos también presentan hipoglicemia sin cuerpos cetónicos. Por lo tanto, esta hipoglicemia es hipo o no cetósica. La hipoglicemia puede ir acompañada de otras alteraciones bioquímicas como hiperamonemia, acidosis metabólica o aumento de las transaminasas simulando un síndrome de Reye.

En algunos pacientes con homocistinuria erróneamente se diagnostica primero un accidente vascular encefálico o un tumor cerebral. Se ha encontrado hemorragia cerebelosa en acidemia propiónica, metilmalónica o isovalérica. La aciduria glutárica tipo I presenta episodios 
de encefalopatía con gran descompensación metabólica y signos extrapiramidales en relación a infecciones virales o gastrointestinales y en un niño que presenta macrocefalia.

Enfermedades como los defectos del ciclo de la urea, las acidurias orgánicas o los defectos de cadena respiratoria puede tener primero anorexia persistente, vómitos recurrentes o episodios de ataxia, retardo progresivo del desarrollo o mal incremento ponderal. Las acidurias orgánicas también presentan algunas veces episodios de deshidratación inexplicada o alteraciones hematológicas con anemia, trombopenia e infecciones intercurrentes. Episodios recurrentes de quetoacidosis pueden ocurrir en la enfermedad de la orina olor a jarabe de arce o en acidosis láctica ocasionada por el déficit de piruvato carboxilasa, en defecto de enzimas de la gluconeogénesis o en diabetes mellitus. Es fundamental medir glicemia, amonio y ácido láctico para poder hacer una aproximación diagnóstica.

\section{Síntomas crónicos y progresivos}

Las enfermedades metabólicas que tienen aparición tardía muchas veces presentan síntomas previos a esta crisis, que pueden pasar inadvertidos. Los síntomas son digestivos, neurológicos y musculares.

Los síntomas digestivos básicamente son anorexia, vómitos y retardo del crecimiento e infecciones frecuentes. Los defectos de la mucosa intestinal pueden ser indistinguibles de acidurias orgánicas, defectos del ciclo de la urea, enfermedades mitocondriales o la intolerancia a la proteína lisinúrica.

Los síntomas neurológicos incluyen retardo del desarrollo psicomotor, convulsiones y alteraciones neurológicas centrales o periféricas. La más frecuente en este grupo es la fenilquetonuria. Sin embargo, en países que realizan la detección neonatal de esta enfermedad desde hace varias décadas ha desaparecido como causa de retardo mental. No es el caso aún en vastas regiones del mundo que cuentan con estos programas desde hace unos pocos años. En Chile, existe un programa nacional desde 1992, por lo tanto está desapareciendo como causa de retardo mental. Se deben descartar también las acidurias orgánicas, defectos del ciclo de la urea, especialmente citrulinemia, acidosis láctica como defecto de piruvato deshidrogenasa o enfermedades del ciclo de Krebs, enfermedades de la neoglucogénesis como déficit de fructosa difosfatasa o déficit de glucosa-6-fosfatasa. Estas patologías pueden ocasionar grave hipoglicemia que deja secuelas neurológicas. La determinación de la relación láctico/pirúvico y la de cuerpos cetónicos es muy importante en el diagnóstico diferencial de las acidosis lácticas. La hepatomegalia también es un signo importante para el diagnóstico diferencial de estas enfermedades.

Síntomas musculares como hipotonía, debilidad muscular o disminución de la masa muscular son frecuentes en las acidurias orgánicas, defectos del ciclo de la urea y en las enfermedades de la cadena respiratoria.

Síntomas específicos y permanentes característicos de un error innato del metabolismo, como cardiomiopatía, hepatomegalia, luxación del cristalino, dismorfias

Cardiomiopatía se puede encontrar en defectos de oxidación de ácidos grasos, enfermedad de Pompe y en las acidurias orgánicas. En la tirosinemia ocurre hiperqueratosis. La tricorrexis nodosa se puede encontrar en la aciduria argininosuccínica, argininemia, intolerancia a la proteína lisinúrica y en la enfermedad de Menkes.

Las enfermedades de depósito lisosomal pueden presentar visceromegalia en los primeros meses y años de vida, como gangliosidosis GM1, enfermedad de Gaucher, enfermedad de Niemann Pick y enfermedad de Wolman. Los niños con mucopolisacaridosis rara vez presentan visceromegalia en los primeros meses de vida. Recién nacidos con hepatoesplenomegalia, facies tosca, hernias y alteraciones esqueléticas e incluso hidrops fetal pueden corresponder a gangliosidosis GM1, mucolipidosis o sialidosis. En estos casos se debe determinar mucopolisacáridos y oligosacáridos en la orina.

Por mucho tiempo se consideró que los errores innatos del metabolismo no presentaban dismorfias. Sin embargo, hoy se sabe que hay algunas de estas enfermedades que presentan un patrón típico de dismorfias, lo que sugiere que la alteración metabólica podría afectar en 
Tabla 2. Muestra crítica

$\begin{array}{ll}\text { Muestra } & \text { Conservación } \\ \text { Plasma 2-3 ml } & \text { Congelar a }-20^{\circ} \mathrm{C} \\ \text { GSPF (gotas de sangre papel filtro) } 4-6 & \text { Secar a } \mathrm{T}^{\circ} \text { ambiente, guardar en bolsa plástica y congelar a }-20^{\circ} \mathrm{C} \\ \text { Orina 20-30 ml } & \text { Congelar a }-20^{\circ} \mathrm{C} \\ \text { LCR } 1 \mathrm{ml} & \text { Congelar a }-20^{\circ} \mathrm{C} \\ \text { Post mortem: biopsia de hígado y músculo } & \text { Congelar a }-70^{\circ} \mathrm{C}\end{array}$

el útero el desarrollo fetal. A este grupo corresponden las enfermedades peroxisomales, por ejemplo la enfermedad de Zellweger o la adrenoleucodistrofia neonatal. En ellas se encuentra hipotonía, epicanto, fontanela amplia, líneas simianas y quistes renales. En la aciduria glutárica tipo II se encuentra una frente amplia, hipertelorismo, orejas de implantación baja, defectos de la pared abdominal, riñones grandes, hipospadias. En el síndrome de Smith-LemliOpitz, de herencia autosómica recesiva y debido a un defecto en la síntesis de colesterol que produce gran aumento de 7-dehidrocolesterol, las dismorfias encontradas son paladar hendido, cardiopatía congénita, hipospadias, polidactilia y sindactilia.

La hiperglicinemia no cetótica presenta agenesia del cuerpo calloso y alteraciones de migración neuronal. El déficit de PDH también presenta agenesia del cuerpo calloso.

La presencia de un olor anormal en la orina o en el cuerpo puede ser el hallazgo más específico en el examen del paciente con error innato del metabolismo. En el niño agudamente enfermo encontraremos olor a azúcar quemada en la enfermedad de la orina olor a jarabe de arce, o a pie sudado en la acidemia isovalérica y en la aciduria glutárica tipo II.

La introducción masiva de la Pesquisa Neonatal Ampliada a 30 patologías metabólicas hace más importante el manejo rápido, pues el pronóstico de estos pacientes depende en gran medida de lo que ocurra en estas primeras horas. Otro aspecto interesante es el hecho que al detectar un niño por Pesquisa Neonatal, muchas veces se encuentra hermanos o parientes con la misma enfermedad, pero que han cursado la historia natural de ella.

Finalmente debemos pensar en la eventualidad de una muerte inminente; se deben tomar las muestras detalladas en la tabla 2. Estas muestras permitirán confirmar un diagnóstico para poder entregar consejo genético a la familia, aunque el niño muera (tabla 2).

\section{Referencias}

1.- Saudubray JM, Sedel F, Walter JH: Clinical approach to treatable inborn metabolic diseases: introduction. en Journal of Inherited Metabolic Disease 2006; 29 (2-3): 261-74.

2.- Leonard JV, Morris AA: Diagnosis and early management of inborn errors of metabolism presenting around the time of birth. en Acta Pediatrica 2006; 95 (1): 6-14.

3.- Burton BK: Inborn errors of metabolism: aguide to diagnosis, en Pediatrics 1998; 102: 1-9.

4.- Raghuveer T, Garg U, Graf W: Inborn Errors of metabolism in Infancy and Early Childhood: an Update. en American Family Physicians 2006; 73: 1981-90.

5.- Raimann E, Cornejo V, Jiménez M: Aproximación al diagnóstico y tratamiento de los errores innatos del metabolismo. En: Errores innatos en el metabolismo del niño, Capítulo 2. Editoras Colombo M., Cornejo V., Raimann E. Editorial Universitaria, 2003, pág 4769. 